\title{
Regional non-uniformity of left ventricular wall movement in $\operatorname{man}^{\star}$
}

\author{
R A GREENBAUM, D G GiBSON \\ From the Cardiac Department, Brompton Hospital, London
}

SUMMARY In order to assess the possible importance of regional non-uniformity of left ventricular function, angiograms of 42 normal subjects and 105 patients with coronary artery disease were digitised frame by frame. Regional curvature around the cavity was assessed, only positive curvature being compatible with ellipsoidal geometry. In normal subjects, positive curvature of the anterior wall was shown by all, but on the inferior wall was present in only $12(28 \%)$ at end-diastole and five $(12 \%)$ at end-systole. In patients with ischaemic heart disease, anterior wall curvature was again positive in all but four with apical aneurysm, but positive curvature of the inferior wall was present more frequently than normal, $47(42 \%)$ at end-diastole and $39(36 \%)$ at end-systole. In these patients, positive curvature at end-diastole was associated with reduced wall movement during ejection. Though the pattern of coronary artery involvement was random, there were conspicuous regional differences in the distribution of abnormal wall movement during isovolumic relaxation. Outward movement was found only on the anterior wall. Abnormal inward movement was five times as frequent on the inferior wall as on the anterior.

An ellipsoidal cavity outline is not therefore characteristic of the normal left ventricle and, when present, this configuration is likely to be associated with a reduced amplitude of inferior wall movement. Regional differences of both structure and function can thus be shown in normal and abnormal hearts. They may arise from variation in local fibre architecture. Their presence must be taken into account in interpreting abnormalities of left ventricular function.

Current views of normal left ventricular function are based largely on extrapolation of the properties of single myocardial fibres. Such an interpretation would only be possible if wall structure were homogeneous, cavity shape that of a simple geometrical figure, and regional differences in function absent. Ellipsodal geometry has frequently been used to represent the cavity outline, allowing volumes to be calculated, shape to be assessed as the ratio of long to short axes or eccentricity, 1 and regional wall stress either longitudinal or circumferential to be calculated from wall thickness, cavity pressure, and predicted local curvature. Recent anatomical studies have confirmed that the first of these assumptions is untenable, and that regional differences exist in fibre architecture within the normal left ventricle. ${ }^{2}$ In order to examine the second, we have taken a single fundamental property of an ellipse, that of constancy of the sign of curvature around its perimeter, and have

$\star$ This research was supported by a grant from the British Heart Foundation. investigated the extent to which this condition applies to the left ventricular cavity outline, localising regions where discrepancies occur. By extending these studies to patients with ischaemic heart disease, we have been able to investigate the third assumption, and to examine how the effect of a well-defined process, coronary artery disease, on wall motion is modified by the position of the affected area on the cavity perimeter.

\section{Methods}

CURVATURE

The curvature of a line at any point can be defined as the reciprocal of the radius of the circle intersecting it tangentially. The curvature of a surface can be defined in exactly the same way in terms of two tangential circles perpendicular to one another. For left ventricular endocardium, the two planes usually chosen are orientated circumferentially and longitudinally with respect to the long axis of the cavity. A circle has constant 
curvature all round its circumference, equal to the reciprocal of its radius of curvature, Curvature varies around the perimeter of an ellipse, dependent on the size of its major and minor axes, but, whatever its eccentricity, the sign of curvature remains constant. If the sign of curvature changes, then convex and concave regions are both present. We have adopted the convention, with respect to the left ventricle, that a surface concave towards the cavity has positive curvature, and a convex one has negative curvature. In the present study we aimed to examine the frequency of occurrence and position around the cavity of negative curvature affecting the left ventricular silhouette as shown in the right anterior oblique projection.

\section{ANGIOGRAPHY}

Angiograms of 147 patients with chest pain were studied. In 42 of these, termed normal, coronary arteriography, left ventricular pressures, and ejection fraction were normal. These patients were described as "normal", though it is recognised that minor or hitherto undescribed abnormalities of left ventricular function or wall motion might have been present. In the remaining 105 patients, the coronary arteriograms, as assessed by an independent radiologist showed significant coronary artery disease to be present, which was classified according to the major vessel affected, a stenosis of 70 per cent or more being regarded as significant.

Left ventriculograms in the right anterior oblique projection were performed before coronary arteriography. An injection of $40 \mathrm{ml}$ contrast medium was made into the left ventricle at a rate of $15 \mathrm{ml} / \mathrm{s}$ and cine film exposed at 50 frames/s. Ectopic and post-ectopic beats were not studied. Calibration was by means of a grid exposed at mid-chest level. The resulting angiograms were digitised, ${ }^{3}$ and the following information extracted:

(1) Plots of cavity volume throughout the cardiac cycle, allowing ejection fraction (stroke volume/enddiastolic volume) to be calculated.

(2) Isometric and contour displays of regional left ventricular wall motion were prepared, as previously described. ${ }^{3}$ In this method, the perimeter of the end-diastolic cavity outline is divided into 40 equal sections, starting from the junction between the aortic root and the mitral valve, and proceeding anticlockwise around the apex and free wall to the aortic root again. In each of these $\mathbf{4 0}$ locations, a plot of regional inward movement of endocardium was constructed. The plots were numbered consecutively around the cavity, so that 1 to 19 represented the inferior wall, 20 the apex, and 21 to 40 the free wall. Records were analysed with particular reference to the detection of abnormal endocardial movement occurring between the timing of minimum cavity area and mitral valve opening. Since in the majority of patients, minimum cavity area approximates to the end of ejection, ${ }^{4}$ we have referred to the interval between it and mitral valve opening as isovolumic relaxation. Normally, during this period 0 to $2 \mathrm{~mm}$ of inward movement occurs on the inferior wall and 0 to $6 \mathrm{~mm}$ outward movement on the anterior wall, ${ }^{3}$ the 95 per cent confidence limits of normal, so that values outside this range were identified. In any ventricle showing such abnormal motion during isovolumic relaxation, the number of plots on which it appeared was identified, each plot covering 2.4 per cent of the cavity perimeter, excluding the aortic root. Histograms were constructed showing the frequency with which each segment was involved with abnormal inward or outward movement during isovolumic relaxation over the total sample of patients studied. The significance of any departure from a normal frequency distribution was assessed by the Kolmogorov-Smirnov test.

(3) Regional curvature: since one of the purposes of the study was to detect major departures from ellipsoidal geometry, curvature was assessed qualitatively as positive, zero, or negative. The anterior wall was taken as the position of the perimeter between the aortic root and the apex, and the inferior wall as that between the apex and the border of the mitral ring opposite the aortic root. Curvature was classed as positive if the perimeter was consistently outside the straight line joining the two ends of the segment, reaching a maximum of $5 \mathrm{~mm}$ or more, and negative if it was consistently inside, reaching $5 \mathrm{~mm}$ (Fig. 1). If the perimeter did not reach $5 \mathrm{~mm}$ or crossed it, curvature was classified as zero or indeterminate. Observations were made on frames corresponding to enddiastole and minimum cavity area.

Attempts at devising algorithms to derive curvature continuously around the perimeter directly from the original data proved unsatisfactory because of minor noise in the digitising process. This could have been overcome by smoothing, but would have required interaction by the observer to confine the process to anterior and inferior walls, and to avoid, for example, inclusion of the apex. Though the final result would probably have been little different from the qualitative process we finally adopted, the complexity of its derivation with increased possibility of error would have reduced the conviction of any conclusions based on its use.

(4) Overall amplitude of movement during ejection, which was taken as ending with minimum cavity area, was assessed as normal, inferior, or 
anterior hypokinesis, when regional wall motion was reduced or absent, or aneurysm. Generalised hypokinesis was diagnosed only when ejection fraction was less than 50 per cent, and the pattern of wall motion symmetrical. When these conditions were satisfied, anterior and inferior hypokinesis were assemed to be present.

Fisher's exact probability test, $\chi^{2}$, and $t$ tests were used to assess statistical probabilities, as appropriate.

\section{Results}

\section{(1) CURVATURE}

In all 42 normal subjects, curvature of the anterior wall was positive throughout ejection. Observations of curvature of the inferior wall are given in Table 1. In only 12 at end-diastole, and five at endsystole was positive curvature of the inferior wall present. In the remainder, it was either negative or zero (Fig. 1).

In the patients with ischaemic heart disease, anterior wall curvature was positive in all but three who had apical aneurysms. In these latter patients, there was an area of negative curvature interposed

Table 1 Curvature of inferior wall at end-diastole and minimum cavity area in 42 normal subjects

\begin{tabular}{llc}
\hline $\begin{array}{l}\text { Inferior wall } \\
\text { curvature }\end{array}$ & End-diastole & $\begin{array}{l}\text { Minimum cavity } \\
\text { area }\end{array}$ \\
\hline Positive & 12 & 5 \\
Negative & 15 & 23 \\
Zero & 15 & 14 \\
\hline
\end{tabular}

between the positive curvature of the upper free wall and that of the aneurysm itself. Curvature of the inferior wall was positive in 47 cases at end-diastole (Table 2), negative curvature occurred in 26 , and it was zero or indeterminate in 32 . At minimum cavity area 39 of those with ischaemic heart disease had positive curvature, significantly more than normal subjects $(p<0.01), 48$ had negative curvature, and 18 had zero or indeterminate curvature. When curvature at end-diastole was correlated with subsequent wall motion during ejection, it was apparent that positive curvature was associated with reduced amplitude of wall motion in 32 cases, and with normal amplitude in 15. Conversely, when end-diastolic curvature was negative, the amplitude of movement was normal in 19 and abnormal in only three. Negative end-diastolic curvature at the border of a discrete apical aneurysm in four patients was not considered in this analysis. These differences are significant $\left(\chi^{2}=7 \cdot 5, \mathrm{p}<0.01\right)$.

Table 2 Relation of amplitude of wall movement to inferior wall curvature in 101 patients with ischaemic heart disease. Four patients with discrete apical aneurysm were not included in this analysis

\begin{tabular}{lllc}
\hline $\begin{array}{l}\text { Inferior wall curvature } \\
\text { at end-diastole }\end{array}$ & \multicolumn{2}{l}{ Amplitude of wall movement } \\
\cline { 2 - 3 } & Normal & Reduced \\
\hline Positive & 47 & 15 & 32 \\
Negative & 22 & 19 & 3 \\
Zero & 32 & 14 & 18 \\
\hline
\end{tabular}

Note: In patients with ischaemic heart disease, hypokinesis is more frequent with positive curvature at end-diastole $(p<0.01)$.

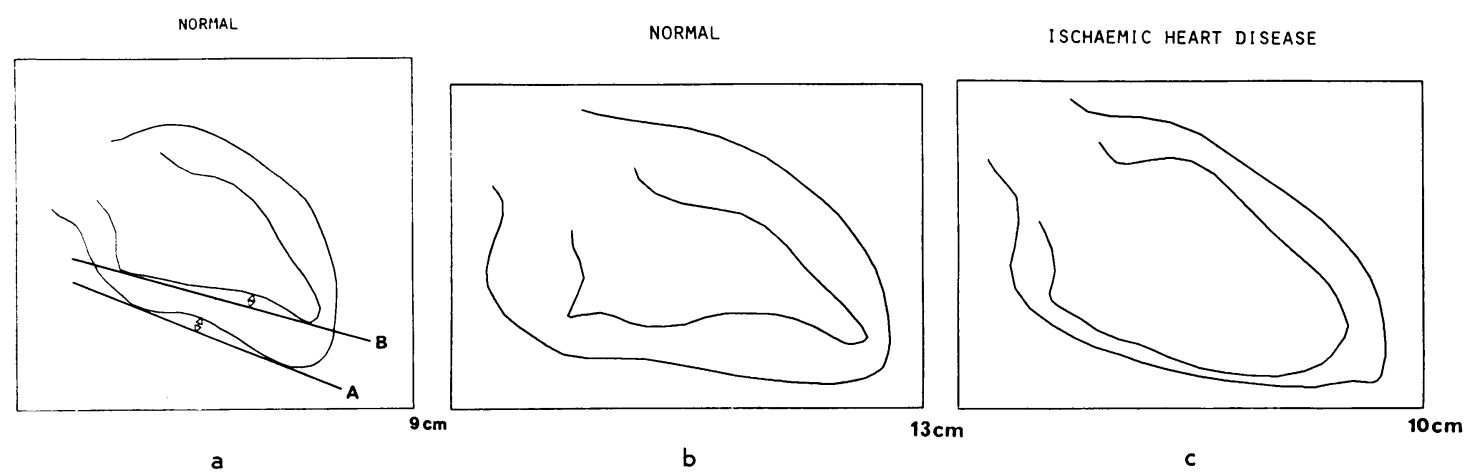

Fig. 1 Representative plots of left ventricular cavity outline at end-diastole and minimum cavity area. (a) Normal subject with negative curvature of the inferior wall in both frames. (b) Normal subject with zero curvature at end-diastole and negative curvature of the inferior wall at minimum cavity area. (c) Subject with ischaemic heart disease showing positive curvature of the inferior wall in both frames. Anterior wall curvature is positive in all cases. 


\section{(2) RELAXATION ABNORMALITIES}

These were present in 15 normals and 47 patients with coronary artery disease. The distribution of early outward movement before mitral valve opening is shown in Fig. 2. Altogether 221 plots were involved. The distribution around the cardiac perimeter was not uniform, but they were confined to plots 21 to 40 , that is the free left ventricular wall between the apex and the aortic root. Within this region, they were distributed as a single normal frequency curve. By contrast, inward movement during isovolumic relaxation occurred more commonly along the inferior wall (Fig. 3), being present in 183 plots, and less commonly on the anterior (34 plots). The distribution of this pattern of wall motion was bimodal, differing significantly from a single normal frequency curve $(p<0.01)$. There was no relation between inferior wall curvature at either end-diastole or minimum cavity area and the presence or absence of abnormal inward movement on this wall during isovolumic relaxation.

(3) PATTERN OF CORONARY ARTERY INVOLVEMENT

In total the left anterior descending was involved 94 times, the circumflex 58 times, and right coronary artery 62 times. The inferior wall is supplied by right and circumflex coronary arteries, and in all but five cases either one or other, or both were involved. The free wall is supplied by the left anterior descending, which was involved in all but 11. Among those with relaxation abnormalities the left anterior descending was involved 41 times,

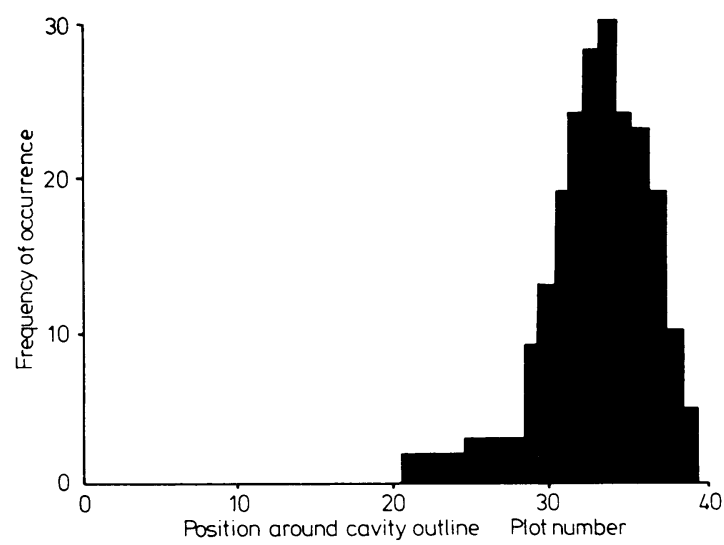

Fig. 2 Histogram of location of plots showing abnormal outward movement of the endocardium during isovolumic relaxation. Abnormal movement is defined as more than $6 \mathrm{~mm}$ of outward movement before mitral valve opening. the circumflex 28 , and the right coronary artery on 32 occasions. This pattern of involvement did not differ significantly from random.

\section{Discussion}

The left ventricle is traditionally viewed as an ellipsoid of revolution, truncated in the region of the mitral ring. Though departures from any simple geometrical shape are naturally to be expected in individual cases, it is perhaps surprising that such a major discrepancy should be overlooked, involving as it does a reversal of the sign of curvature rather than minor deviations in its magnitude, and affecting more than one-third of the cavity outline. Regional configuration of cadaver hearts has previously been measured by Hutchins et al. ${ }^{5}$ who found long axis curvature to be uniformly positive. These authors, however, made their apex to base measurements along the free wall at the level of minor dimension in a region where we also found positive curvature. Flattening of the inferior wall was described by Grant in $1953,{ }^{6}$ who attributed it to deformation of the heart by the diaphragm, but its persistence during ejection makes this an unlikely explanation. Negative curvature cannot be regarded as abnormal, since it occurs more commonly in patients with normal haemodynamics, left ventricular cavity size, ejection fraction, and coronary arteriograms than in those with coronary artery disease. Even in the latter group of patients, its presence is more commonly associated with normal wall motion and its absence with regional hypokinesis during ejection.

Negative curvature affecting so large a portion of the cavity has a number of implications in the

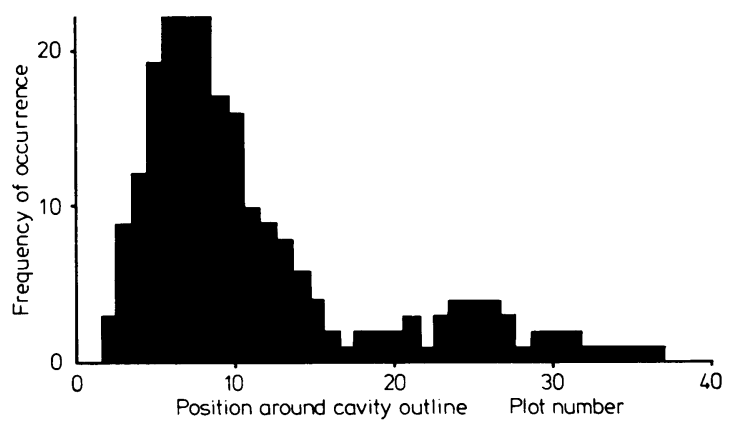

Fig. 3 Histogram of location of plots showing abnormal inward movement of endocardium during isovolumic relaxation. Abnormal movement is defined as more than $2 \mathrm{~mm}$ of inward movement before mitral valve opening. 
study of left ventricular function. Regional wall stress is usually calculated using Laplace's relation or one of its variants. With this approach, however, negative curvature in the longitudinal direction will result in calculated systolic wall stress also being negative, which seems unlikely. This underlines that negative curvature may have potential functional significance and shows the limitations of applying Laplace's law to a structure such as the left ventricle in which cavity pressure is secondary to active tension within the wall and not the reverse. Overall wall stress at any point depends on the sum of circumferential and longitudinal curvature. If curvature is positive in one plane and equal but negative in the other, the resulting surface is a catenoid, for which a net zero stress is predicted regardless of the pressure within the lumen. This configuration has been described as applying to the interventricular septum in hypertrophic cardiomyopathy, ${ }^{7}$ and the suggestion made that the resulting dissociation between cavity pressure and wall stress is responsible for inappropriate hypertrophy in this disease. The frequent occurrence of similar conditions in the inferior wall of the normal heart, however, must shed some doubt on this suggestion. Negative curvature developing or persisting during ejection is also a significant cause of the characteristic elongation of left ventricular cavity shape at end-systole, which is a feature of normal left ventricular function. If left ventricular shape changes are to be followed during the cardiac cycle, therefore, it seems appropriate to use a means of assessing them which does not presuppose any idealised geometry, ${ }^{8}$ rather than those based on an ellipse. The persistence of negative curvature during ejection also gives information about local myocardial fibre orientation. Shortening of muscle fibres parallel to the long axis of the ventricle would cause regions of endocardium showing negative curvature at end-diastole to move outwards and not inwards with tension development, a mechanism that has been invoked to explain reversed septal motion in the presence of right ventricular overload.9 Observation of regional curvature of the inferior wall thus shows that normal left ventricular shape is associated with major departures from ellipsoidal geometry, and that these are of a type that have potential structural and functional significance.

Further evidence that regional wall motion might be affected by position in the wall rather than directly by underlying disease came from observations made in patients with ischaemic heart disease. In the sample we studied, the pattern of coronary artery involvement did not differ from random. In spite of this, abnormalities affecting the inferior wall were very different from those seen on the anterior. On the inferior wall, only one type of abnormal wall motion was seen, consisting of prolonged inward movement of endocardium. This also involved the anterior wall, though less frequently. Previous studies have shown that this behaviour is commonly associated with impaired systolic performance in the same region, and, when present in single vessel coronary artery disease, is frequently in the territory of the affected vessel. ${ }^{3}$ In the present study, the frequency of its appearance was not directly related to that of the coronary artery disease, since it occurred five times as frequently on the inferior wall as the anterior, though the pattern of coronary artery involvement was similar. When early outward movement of endocardium during isovolumic relaxation was considered, the discrepancy became even more striking, since this abnormality was not found to involve the inferior wall in any instance. We conclude, therefore, that the distribution of abnormal wall motion during isovolumic relaxation cannot be explained only in terms of the pattern of coronary artery involvement, and that an additional "local" factor must be taken into account.

Regional differences in shape and function of the normal left ventricle have received little attention. It has recently been pointed out, however, that the peak velocity of inward movement of the inferior wall during ejection is approximately half that of the anterior ${ }^{10}$ and similar differences have been noted in amplitude. ${ }^{11}$ During isovolumic relaxation, the upper limit of normal of outward movement of the anterior wall is approximately five times that of the inferior. ${ }^{3}$ The pattern of rapid wall thinning in normal children also shows striking regional variation, being virtually absent in the septum, and present to its maximum extent in the mid-portion of the free wall with intermediate values seen in the free wall towards the base of the heart. ${ }^{12}$ In ischaemic heart disease also, an unexpected localisation of abnormalities of isovolumic contraction to the inferior wall has been described, which is also not explicable on the basis of the pattern of coronary artery disease, and apparently identical abnormalities may occur in patients with chest pain and normal coronary arteriograms. ${ }^{13}$ We suggest that these phenomena be described as "regional non-uniformities" of structure or function, in order to stress that they may be a normal finding, and to distinguish them from contraction or relaxation abnormalities that occur as the result of local disease.

We have no direct evidence for the mechanisms underlying this regional non-uniformity. The accentuation of negative curvature on the inferior 
wall when prolonged inward movement occurs, and the reverse with abnormal outward movement during isovolumic relaxation both suggest that abnormalities of wall motion at this stage in the cardiac cycle are closely associated with regional patterns of curvature. It seems unlikely that local differences in the underlying contractile process occur, and, for reasons discussed above, enddiastolic cavity shape itself is not the only mechanism. A much more probable explanation is that regional differences in shape and function reflect corresponding variation in myocardial fibre architecture. The free wall of the left ventricle seen angiographically corresponds to the obtuse margin at the level of the papillary muscles. In this region, both epicardial and subendocardial fibres are arranged longitudinally, while in the mid-wall, there is a well-developed ring of circumferential fibres. ${ }^{2}$ On the diaphragmatic aspect of the left ventricle, corresponding to the inferior wall seen angiographically, extensive regions of negative curvature can also be shown anatomically. Fibre structure here differs in that the subepicardial fibres are arranged circumferentially near the apex and crux, and obliquely in between. In addition, the inferior wall viewed angiographically overlaps the area from which "cross-over" fibres arise from the subepicardial and subendocardial layers to give rise to the interventricular septum. ${ }^{2}$

This relative lack of longitudinal fibres is compatible with the functional considerations described earlier. It is possible that right ventricular function might have contributed directly to these abnormalities of the inferior wall, but this seems unlikely, since curvature was negative in patients in whom right ventricular pressures were normal, and positive in those with left ventricular disease, in whom they would be expected to be raised. The extent to which myocardial architecture may be affected in ischaemia has not been determined, though it seems likely that the phenomenon of early outward movement is particularly dependent on the integrity of local fibre structure, ${ }^{14}$ and might thus be lost with infarction or fibrosis.

We conclude, therefore, that regional nonuniformity is a relatively common finding in patients with normal or abnormal hearts. Local differences in cavity outline, endocardial motion, or wall dynamics cannot always on their own be taken as evidence of disease but may be manifestations of the normal, highly organised processes of ejection and filling. We feel that these phenomena are worthy of further study, not only for the light they may shed on normal function and possibly structure, but also as additional factors that must be taken into account in elucidating mechanisms of left ventricular disease.

\section{References}

1 Vokonas PS, Gorlin R, Cohn PF, Herman MV, Sonnenblick EH. Dynamic geometry of the left ventricle in mitral regurgitation. Circulation 1973; 48: $786-96$.

2 Greenbaum RA, Ho SY, Gibson DG, Becker A, Anderson RH. Left ventricular fibre architecture in man. $B r$ Heart $\mathcal{F}$. In press.

3 Gibson DG, Prewitt TA, Brown DJ. Analysis of left ventricular wall movement during isovolumic relaxation and its relation to coronary artery disease. Br Heart f 1976; 38: 1010-9.

4 Gibson DG, Marier DL. Limitations of the two frame method in displaying left ventricular wall motion (abstract). Circulation 1979; suppl II: 262.

5 Hutchins GM, Bulkley BH, Moore GW, Piasio MA, Lohr FT. Shape of the human cardiac ventricles. Am 7 Cardiol 1978; 41: 646-54.

6 Grant RP. Architectonics of the heart. Am Heart $\mathcal{f}$ 1953; 46: 405-31.

7 Hutchins GM, Bulkley BH. Catenoid shape of the interventricular septum: possible cause of idiopathic hypertrophic subaortic stenosis. Circulation 1978; 58: 392-7.

8 Gibson DG, Brown DJ. Continuous assessment of left ventricular shape in man. Br Heart $\mathcal{F}$ 1975; 37: 904-10.

9 Pearlman AS, Clark CE, Henry WL, Morganroth J, Itscoitz SB, Epstein SE. Determinants of ventricular septal motion: influence of relative right and left ventricular size. Circulation 1976; 54: 83-91.

10 Gibson DG, Sanderson JE, Traill TA, Brown DJ, Goodwin JF. Regional left ventricular wall movement in hypertrophic cardiomyopathy. Br Heart $\mathcal{f}$ 1978; 40: 1327-33.

11 Ingels NB, Jr, Daughters GT II, Stinson EB, Alderman EL. Is wall motion in the normal left ventricle uniform, symmetric and synergic? Circulation 1979; 60, suppl II : 93.

12 Marier DL, Gibson DG, St John Sutton MG. Regional variation in posterior wall dynamics in normal children (abstract). Circulation 1979; 60, suppl II: 92.

13 Gibson DG, Doran JH, Traill TA, Brown DJ. Abnormal left ventricular wall movement during early systole in patients with angina pectoris. $\mathrm{Br}$ Heart f 1978; 40: 758-66.

14 Gibson DG, Greenbaum R, Marier DL, Brown DJ. Clinical significance of early diastolic changes in left ventricular wall thickness. Eur f Cardiol 1980; suppl A: 157-63.

Requests for reprints to Dr D G Gibson, Cardiac Department, Brompton Hospital, Fulham Road, London SW3 6HP. 